\title{
Subject-formal Methods Based on Cognitive Maps and the Problem of Risk Due to the Human Factor
}

\author{
Abramova N., Avdeeva Z., Kovriga S. and Makarenko D. \\ Institute of Control Sciences of Russian Academy of Sciences \\ Russian Federation
}

\section{Introduction}

Recently there appear more and more publications, both theoretical, and applied related to cognitive maps, though with different meanings of "cognitive map" by different authors.

Contemporary cognitive map applications cover different domains: economics, medicine, foreign affairs and others. The spectrum of the problems solved spreads from conceptual modeling aimed to help individual to better structure and understand the problem, up to deriving a shared understanding of the problem, then to most typical simulation of illstructured situations optionally including their dynamics, and finally to solution of some strategic management problems.

In theoretical studies related to cognitive maps we discriminate two fundamental approaches different in research aims: normative (as people should think) and descriptive (as people do think). Hereby we apply the idea suggested by A. Tversky et al. and presented in the widely known book (Bell et al., 1988) to cognitive map researches.

Followers of the descriptive approach, investigating cognitive processes in people, while decision-making, refer to the concept of cognitive map ${ }^{1}$ as the internal model of person's knowledge about some situation².

This interpretation to some extent correlates with the definition from Wikipedia ${ }^{3}$, though to our opinion it would be more correct to speak about "mental representations composed as a result of a series of psychological transformations" instead of "mental processing composed of a series of psychological transformations".

In the normative approach which applied aim is to suggest people the ways of practical problem solution, cognitive maps of various kinds are proposed as normative models (in other words, as schemes or rules) for external representation of knowledge about situations (whatever the internal representation could be). In this approach the cognitive map of a concrete situation has an external form of structure consisting of elements (named concepts,

\footnotetext{
${ }^{1}$ Other terms like mental maps, cognitive models, or mental models are often used as well.

2 which can be visualized.

${ }^{3}$ http://en.wikipedia.org/wiki/Cognitive_map

Source: Cognitive Maps, Book edited by: Karl Perusich,

ISBN 978-953-307-044-5, pp. 140, January 2010, INTECH, Croatia, downloaded from SCIYO.COM
} 
factors, or constructs) and, as a rule, causal ${ }^{4}$ relations (or links) between these elements and sometimes with some additional attributes (signs, weights of influences).

In cognitive-map-based approach to searching and making decisions, maps with different degree of formalization are used, thus providing the possibility of more or less complicated formal methods application (For an overview see Kuznetsov et al., 2006; Pena et al., 2007; Abramova et al., 2008).

This work is oriented to formal cognitive maps and associated techniques for searching and making decisions in control of complex and ill-structured situations.

There is at least one problem that calls forth involving descriptive approach knowledge into the field of formal models and methods for complex and ill-structured situations (with cognitive maps or not). This is, namely, the problem of risks due to the human factor in this field (Abramova, 2007; Abramova \& Kovriga, 2008). First of all the problem lies in the inevitable and substantial humans' participation in solving practical problems with formal methods (at least for formalization of primary representations of a situation), so that it is pertinent to speak about subject-formal instead of formal methods. Due to the humans' participation such methods basically cannot provide validity ${ }^{5}$ of ultimate results, or in other words, they are basically risky concerning results validity.

The pragmatic importance of the given problem of risks obviously depends on how much significant are the risks to obtain invalid results in solving practical problems. By present time theoretical, experimental and even practical knowledge has been accumulated, leading to understanding or directly saying that human factors can be a significant source of risk for the results validity (Abramova \& Kovriga, 2008). However the importance of the problem in the field of formal decision-making methods is underestimated by scientific community, despite some widely known researches such as the ones on "logic of failure" by D. Dörner (Dorner, 1997) and on psychological correctness in the formal theory of decision-making by O. Larichev and his school (Larichev et al., 1997). (In some more detail see (Abramova, 2007; Abramova \& Kovriga, 2008)). This fully concerns the cognitive-map-based subject-formal methods.

The problem of human-induced risks in solving practical control problems by means of subject-formal methods and computer-aided technologies (with cognitive mapping being just a special case in the field) is only recently put forward as the complex problem covering the whole life-cycle of such methods and technologies (Abramova, 2007, Abramova \& Kovriga, 2008), and it almost has not been explored yet.

In our research of risk factors and ways the human-induced-risk problem might be solved, the interdisciplinary approach is accepted, following the prescriptive approach (in terms of (Bell et al., 1988)) with the idea of integrating normative and descriptive knowledge in decision making but going much further in the scientific knowledge integration with involving relevant knowledge from linguistics, cognitive sciences, sociology, artificial intelligence, computer science, philosophy.

In (Abramova, 2007) a number of principles of protection against human-induced risks at all stages of the subject-formal method life cycle, starting from verification of theoretical

${ }^{4}$ or, that is the same, cause-effect

${ }^{5}$ Note that validity of results of a method application is understood here in wide intuitive sense as capability to rely upon these results in solving a specific practical problem. It is also possible to speak about validity of a method as its capability to yield valid results. 
background and justification of a given method and finishing with verification of formalized expert knowledge and beliefs, have been proposed and is being practically tested (Abramova, 2007). The principles cover various theoretical models of expert knowledge about a problem situation, including formal cognitive maps.

Our principles are well agreed with the known ideas by R. Heuer (Heuer, 1999) about the cognitive challenges intelligence analysts face in the complex information processing, and with his program for "a prudent management system", which, in our terms, proposes verification of analysts' knowledge and reasoning. However, essential difference of our approach consists in focusing on formal methods which can be used by analysts and experts, and not only these people are considered as risk sources, but also theorists, and developers of information technologies and decision-support systems.

In publications concerning cognitive maps (mainly descriptive ones), and also in the adjacent areas one can find some useful information, relevant to the problem of risks due to the human factor using cognitive-map-based methods. Except the works mentioned above (Larichev, 1997; Dorner, 1997; Abramova, 2007), which relation to the problem of risks is briefly described in (Abramova \& Kovriga, 2008), the significant results on risk factors and suggested ways of their overcoming can be found in (Heuer, 1999; Hogdkinson et al., 2004; Schaffernicht, 2007).

The present chapter contains: (1) a short survey of contemporary cognitive maps (section 2.1 ); (2) some ideas relevant to the problem of risks due to the human factor in cognitivemap-based methods (section 2.2); (3) description of two interdisciplinary models, that allow analysis, explanation and forecast of human-induced risk factors in the life cycle of subjectformal methods (section 3); (4) recently discovered human-induced risk factors that reduce validity of cognitive-map based subject-formal methods (section 4); (5) some principles to reducing the risks due to the human factor, and a number of heuristic criteria of validity of use of cognitive maps, applicable for early detection of direct semantic errors and risks of formalization (section 5).

Some of the discovered risks are demonstrated with cognitive maps found in the scientific literature as well as maps that have been used to solve real control problems.

\section{Short review of contemporary formal cognitive maps. Some ideas relevant to the problem of human-induced risks in cognitive-map-based methods}

In modern practical and theoretical works the concept of "cognitive map» is used more and more in different fields, but it takes various meanings. Often instead of cognitive maps it is spoken about causal maps (Narayanan \& Deborah, 2005). Schemes (circles of influence, Ishikava diagrams, strategic maps, etc.) reflecting cause and effect aspects of a business situation while solving strategic planning problems are also referred to as cognitive maps according to some attributes (Bouzdine-Chameeva, 2006). In some works cognitive maps are reckoned among cause and effect schemes where certain logic or mathematical feature of cause and effect relations is reflected (Schaffernicht, 2007), or among conceptual maps with the fixed type of relations (causal) (Huff, 2005 is reflected).

The spectrum of problems in the field of solving ill-structured problems, where it is suggested to use cognitive maps, depends on the kind of maps, and substantially, from the degree of their formalization. 
Let us systematize the contemporary cognitive maps and assign the ones of them which are used as the basis for development of subject-formal methods for solving the problems of forecast and control of ill-structured objects and situations.

\subsection{Comparative analysis of cognitive maps with regard to formalization degree}

In this work the term "cognitive map" refers to the family of semi-formalized 6 and formal models representing the structure of causal (or, that is the same, cause-effect) influences of a mapped ill-structured situation. Among such cognitive maps we can separate semi-formalized cognitive maps (Eden et al., 2005; Huff, 2005; Narayanan \& Deborah, 2005 for an overview), applied for forming general concept of a situation and analysis (comparison) of person's viewpoints regarding a situation and formal cognitive maps applied for analysis and modeling of ill-structured situations on the basis of individuals' beliefs. Formally, the obligatory base of all models is a directed graph, which nodes are associated with elements (short phrase for weak-formal ones and concept, variables or factors for formal map) and arches are interpreted as direct causal influences (or causal relations, connections, links) between factors. Usually the obligatory base is added with some parameters, such as an influence sign ("+" or "-"), including for weak-formal cognitive map, or influence intensity, and some other interpretations for formal cognitive maps both substantial, and mathematical are given to the map. In semi-formalized maps there are no any other formalization except graph. At that, the methods of analysis and comparison of the maps on the base of the graph theory are developed.

Among different maps with different degree of formalization, beginning from the fundamental woks by R. Axelrod and F. Roberts (Axelrod, 1976; Roberts, 1976), the one can separate a family of maps which can be appropriately called as formal maps. Their distinctive feature are the theoretical models that describe the semantics of this or that map type, that makes them computational (in terms of (Kremer, 1994)) or executable concept-models ((in terms of (Schaffernicht, 2007)) and affords obtaining new knowledge with formal methods (such as simulation, inference).

With regard to the described situations, one can separate static and dynamic cognitive maps. Semantics of the static cognitive maps does not contain time aspect (earlier-later).

To formal cognitive maps correspond to theoretical (general) model which include the formal description of a map, and also obvious or implicit influences aggregation function on the factor. Various interpretations of nodes, arcs and weights on the arcs, as well as various influences aggregation functions onto factors result in various types of theoretical model of cognitive maps (or cognitive map types) and formal means for their analysis.

The general models for different type of maps form schemes of representation of knowledge about concrete situations in the course of formalization. In case when influences aggregation functions onto factors and maps parameters contain the time in an explicit form, such maps will be referred to as maps with strong dynamics. Other cognitive maps will be referred to as the ones with weak dynamics.

The following maps can be referred to the maps with weak dynamics: fuzzy cognitive maps of Cosco (Khan et al., 2004; see the review of maps of this kind in Kuznetsov et al., 2006; Vesa et al., 2007), logic cognitive-map-based on the relational algebra (Axelrod,1976; Chaib-

${ }^{6}$ following of the Kremer's terminology 
draa, 2002; Pena, 2007) or other model (Chen, 1995), Wellman probabilistic cognitive maps (see review in Pena, 2007), and others.

The cognitive maps with strong dynamics are classified by the type of the node aggregation function, for example, a linear function is introduced for the maps in the spirit of F. Roberts, a fuzzy function is applied for the dynamic maps in the spirit of Cosco (Silov, 1995; Fedulov, 2005). The concrete map with set of parameters ${ }^{7}$ describes not a single situation and not a single dynamic process, but a variety of the processes different in parameters.

To distinguish between current situations and dynamic processes generated by them in the course of modeling, it is accepted to speak about initial conditions. Accordingly in the language of modeling for description of concrete situations and problems being solved we may separate the language of cognitive maps and language of initial conditions so the concrete cognitive map together with initial conditions sets model of a concrete situation.

Let us consider in detail the cognitive maps in the spirit of Roberts, that form a family of general models of cognitive maps with strong dynamics with base linear impact model, offered by Roberts for the sign and weighed graphs (corresponding to maps) for solving the problems of forecasting of complex system behavior. (Roberts, 1976). ${ }^{8}$ For such maps the type of the aggregation function for causal and externally conditioned influences on factor $x_{i}$ is defined as follows:

$$
x_{i}(t+1)=x_{i}(t)+\sum_{j \in I_{i}} a_{i j}\left(x_{j}(t)-x_{j}(t-1)\right)+g_{i}(t), \quad i=1, \ldots, N
$$

where $x_{i}(t+1)$ and $x_{i}(t)$ are the values of $i$-th factor at instants $t+1$ and $t$, respectively, $x_{j}(t)-x_{j}(t-1)=\Delta x_{j}(t)$ is the increment of factor $x_{j}, a_{i j}$ is the weight of factor $x_{j}$ influence onto factor $x_{i}, I_{i}$ is the set of factors directly effecting factor $x_{i} ; g_{i}(t)$ - external influence at instant $\mathrm{t}$.

Cognitive maps of this type differ with regard to modifications of the base model. Weight anyhow formally is represented in all such maps, for example, by number or linguistic value of type "strongly (poorly) influences" which is automatically converted into number.

In a number of works the base model (1) is projected onto the maps, representable by the functional graph ${ }^{10}$. On the base of such maps there are developed various methods and approaches (Kulba et al., 2004; Gorelova, 2006) that support searching solution in control of ill-structured situations, in particular, the methodology for deriving scenarios of illstructured system that allows carrying out research of its behavior for various control actions. It should be noted that some researchers (Kulba et al., 2004) working with the such models do not use the concept of cognitive map for complex situations model.

One more widely used modification of the maps in the spirit of F. Roberts is the model of cognitive maps of E. K. Kornoushenko and V. I. Maximov ${ }^{11}$ with values of factors and

\footnotetext{
7 i.e., dynamic system from the formal point of view.

${ }^{8}$ Generally speaking, the basic results have been received for sign graphs, and inclusion of weights was considered as model development.

${ }^{9}$ In base model the concept variable, instead of factor is used.

${ }^{10}$ In the specific case, a real number (then it is a weighed graph).

${ }^{11}$ Hereinafter referred to as Kornoushenko-Maximov cognitive maps.
} 
relations from the interval [-1, 1] (Avdeeva \& Kovriga, 2008; Avdeeva et al., 2007; Kornoushenko \& Maksimov, 2001) ${ }^{12}$. This research team uses linguistic variables, which automatically converted to numbers. Another particularity of theoretical model of Kornoushenko-Maximov cognitive maps is the "compulsory" stabilization by proportional change of the map weights (Maximov \& Kornoushenko, 2001). This research group has developed the following subject-formal methods and corresponding information technologies: method of structure and goal analysis of ill-structured system development; approach to studying conflict situations generated by contradictions in interests of subjects influencing development of considered system; approach and methods for solving illstructured problems and deriving scenarios of ill-structured system development.

The formal cognitive maps and the subject-formal methods on their basis are the ground for construction of practical situations models, and then the criterion of the map adequacy is the situation under investigation. It determines the need for taking into account the problem of risks relating mainly to the distortions in formalization of person's initial knowledge.

\subsection{Some ideas relevant to the problem of risks due to the human factor in cognitive- map-based methods}

Among some representatives of the descriptive approach who investigate how professional analysts and beginners think (without necessary direct relation to cognitive maps), the one will notice the interest to the questions of validity of knowledge representation and problem solving for complex situations.

On the contrary, the community of the scientists in the field of formal decision-making theories and specialists implementing formal methods in information technologies, as a rule, rely on common sense regarding representations of the ways the person should think (including both knowledge representation schemes and the order of their filling).

Among few works in the field of cognitive mapping, with recognizing not only the human factor influence, but also necessity of researches in this direction, it is worthwhile to note (Bouzdine-Chameeva, 2006), where validity of cognitive maps with internal validity between the data and the conceptualization of the data, including the definitions of concepts and influences, has been discussed, proceeding from the general ideas of content-analysis reliability. The analysis of publications relevant to the problem of risk has been directed mainly on revealing factors, significant for working out subject-formal methods protected from invalidity of final results.

In the field related to cognitive maps the closest to this topic is the experimental research by G. Hodgkinson at al. (Hodgkinson et al.,2004). It is devoted to rigorous evaluating the relative merits of the alternative knowledge elicitation procedures in causal mapping. In the context of the problem of risks, this research draws attention to the experimentally confirmed fact of essential dependence of resulting maps on the elicitation method (in the experiment, pairwise comparisons technique versus freehand drawing). It is argued that all mapping techniques involve recall and recognition processes to different extents, up to some elements being constructed "online". In that, distortions (in our terms), caused by a mapelicitation-method may have not only more or less negative character (due to recall and recognition errors), but also may develop creative insights.

12 Such modification makes a map nonlinear. 
R. Heuer (Heuer,1999) considers cognitive biases in analysts' judgments and solutions, i.e. mental errors caused by simplified information processing strategies, those being similar to optical illusions in that the error remains compelling even when one is fully aware of its nature. He marks out biases in perception of cause and effect which may be significant at cognitive mapping. (For example, analysts often perceive relationships that do not in fact exist via illusory correlations or tend to assume that economic events have primarily economic causes.)

In (Richardson, 1997) it is shown that in word-and-arrow diagrams with positive and negative signs on links (including dynamic cognitive maps) link is semantically doublemeaning: it can be interpreted both as proportional and additive influence, with the latter meaning for the accumulating nature of rate-to-level link. According to Richardson, the double-meaning in specific situations can be resolved if the modeler knows the meanings of the concepts in the diagram and thus knows necessary meaning of the link. However, as Shafferniht showed in his work with students, people are inclined to simplify the understanding of polarity of influences, and find difficulty in distinction of different kinds of dynamic causal influences, using the simplified ("popular") interpretation of polarity. Moreover, to the above godsends we can add that in formal dynamic maps, such as maps in the spirit of Roberts, the identical semantics of all influences usually proportional or close to it, is implied so indistinct human understanding of influences is meant.

Below (in section 3.1) we show that the discovered problems can be taken into account while analyzing the risks in application of various cognitive-map-based methods.

The question "what should be done" in regard with the discovered difficulties has one of the answers in essence that consists in development of training methods that take into account the cognitive peculiarity of adults and beginners. The most radical answer is given in the program of R. Heuer (Heuer, 1999). This program assumes, for the purpose of increasing the validity of results of intellectual analysis in the complex information processing, the whole complex of long-term measures, including, financial and organizational ones, and a number of mental techniques, some of which are applicable in cognitive mapping, including application of formal cognitive maps. In our terms, he offers verification of analysts' knowledge and reasoning.

\section{Analysis, explanation, and forecasting of risks: models and approaches}

\subsection{The general model of computer-aided decision making}

The present section describes the simplified model of the decision making process by means of computer-aided subject-formal method for ill-structured problem situations. The purpose of this model is to describe and explain one general risk occurring mechanism when using such methods in practical decision-making. The model is also applicable as a prototype to analyze the risks due to the human factor in more complicated decision-making processes. The model represents a decision-making process as search for a solution of a problem situation that consists of 3 stages: 1) forming and formalization of representations and beliefs about the situation which is carried out by decision-maker or decision-making personnel and finishes by entering a formalized situation model into the computer; 2) generation of new formal knowledge about the way the problem situation may be resolved by processing entered information in the computer (analysis, forecasting, search and justification of decisions, etc.); 3) digestion of new knowledge by decision-making personnel to be used to control the situation. The first stage is sometimes interpreted by information technology developers as expert knowledge elicitation or acquisition. 
From the point of view of human induced risk analysis, such a process of the new human knowledge generation is interpreted as a process of initial (internal) person's knowledge generation and its cognitive transformations into the final result which depends on the way of knowledge formal representation in the computer.

The model of the cognitive knowledge transformation process is presented in fig. 1.

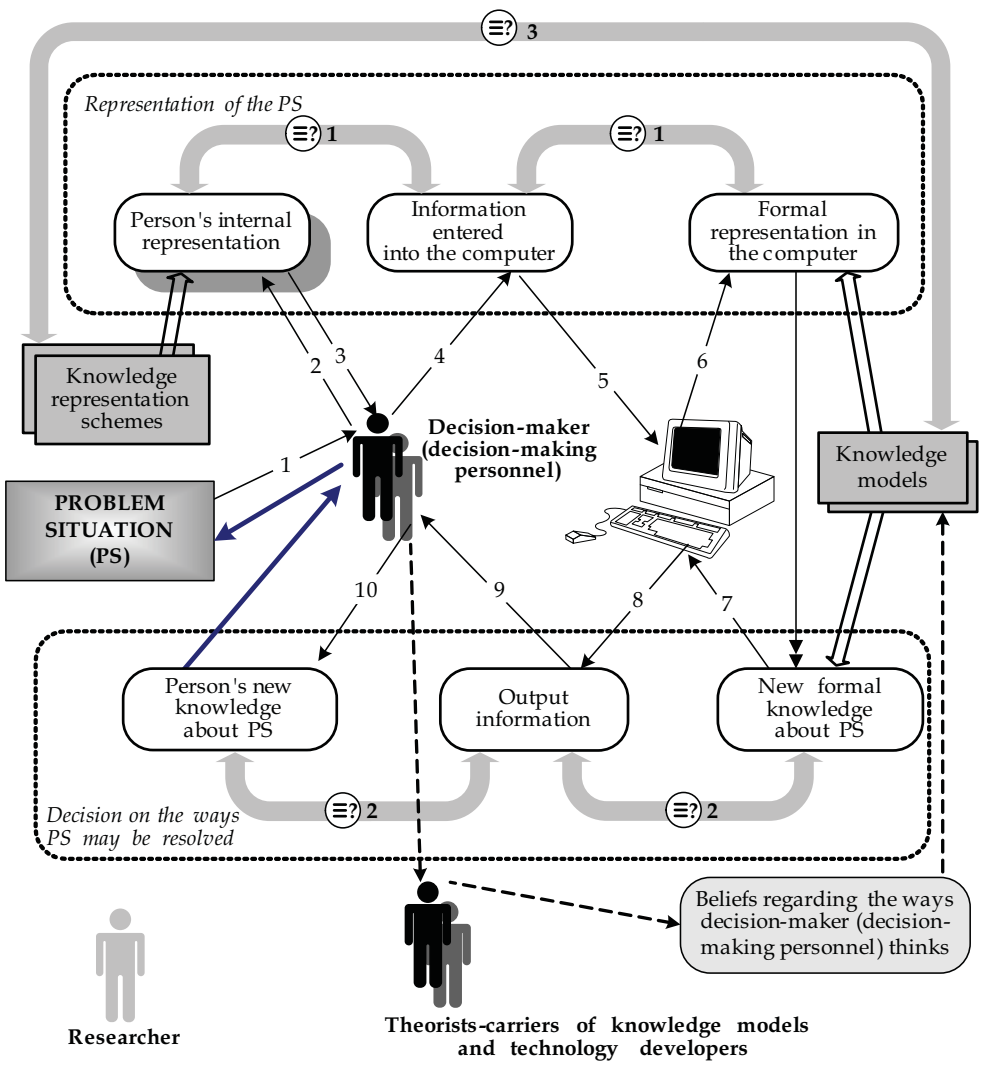

Fig. 1. Model of cognitive transformations while searching and making decision in illstructured problem situation

The model is simplified: in particular, a single decision-maker is considered, so everything concerned the matching of different people's representations and beliefs are omitted; the structure of the process is simplified as much as possible.

Methodologically the transformations are represented in the spirit of V. Lefebvre (Abramova et al., 2009) as reflections of a real-world situation and others' representations about the situation in the person's mind.

In the model in fig. 1, the problem situation is primarily reflected in the person's mind and as a result his internal representation about the situation is formed (it is shown by thin arrows 1 and 2). Then the formalization step, i.e. the secondary reflection takes place, and it finishes with entering the information into the computer (arrows 3 and 4). This information changes its carrier and is being filled with the meaning once again, thus it becomes 
knowledge of "the formal expert", that is the computer (arrows 5 and 6). (Certainly, in a strict sense, the computer only processes the information, however it can be considered as meaningful due to adding a formal sense according to which it is formally processed further.) New formal knowledge about the problem situation and the ways it may be resolved is generated by the computer (double end arrow), and undergoes a similar chain of transformations in a reverse order (arrows 7, 8, 9, 10). As a result the new knowledge is comprehended by the decision-maker, "fitted" to his initial knowledge, and, if acceptable, can be used to influence the problem situation (bold arrows in fig. 1).

The described model of knowledge transformations is in whole (to within simplifications) applicable for a special case of subject-formal cognitive-map-based methods.

When analyzing the above process with risks in view (fig.1), it is necessary to answer the question whether it is possible to consider primary reflection of the initial situation (arrows 1,2 ) and transformations of knowledge "about the same" (arrows 3...6) as acceptable in respect to validity of final results. The similar questions stand for transformations of the results (arrows 7...10). (In fig. 1 these questions are presented as questions of identity of the corresponding representations, marked as " $\equiv ? 1^{\prime \prime}$ and " $\equiv ? 2$ "). In other words, the question is about adequacy or at least acceptability of reflections of knowledge "about the same", from the point of view of the researcher supposed to be objective and informed.

In our early works based on theoretical considerations in various domains of scientific knowledge including philosophy, linguistics, psychology, artificial intelligence, the principle of the distorting effect was put forward that affords to characterize the considered objective processes of reflections with the following theses.

1. Reflection of various objects in the person's mind, in general, happens with distortion.

2. The inevitable source of distortion in the person's reflected knowledge is the person himself with his internal cognitive means.

3. Knowledge transfer between persons, in general, occurs with distortion.

The studies show that as to consequences, distorting effects in knowledge can be considerable and not considerable, positive and negative, acceptable and not acceptable; positive effects promote achieving goals, negative ones prevent it or at least become risk factors.

Let us briefly describe the basic person's cognitive means at primary reflection of a problem situation and the subsequent formalization. It is assumed that the basic person's means at primary reflection are the internal knowledge representation schemes, inherent in a person and frequently unconscious, as well as the general concepts which are jointly structuring his internal knowledge and beliefs about a problem situation.

It is important to underline that in the model there is no assumptions about the structure of internal representations (in the spirit of cognitive map definition from Wikipedia ${ }^{13}$ ), beyond the assumption of dependence on internal cognitive means

At the formalization stage the basic cognitive means are theoretical models of knowledge of the situation and so-called interface concepts of computer technologies (Abramova, 2002), which are usually a component of the above models. These concepts are used to elicit formalized knowledge from problem area experts and to interpret the results generated by computer.

13 http://en.wikipedia.org/wiki/Cognitive_map 
In the case of cognitive mapping with formal maps we, first of all, mean theoretical models of some or other type of maps, models of interaction with the environment, initial conditions to distinguish a current situation in the set of situations described by the given map. The basic interface concepts are the factor (or variable), influence (or link, relation), influence weight (or intensity).

According to the distorting effect principle, both these means and their change in the course of knowledge transformations, become the risk factors. When the computer joins the process of knowledge transformation as "the formal expert", there arises a situation of change of knowledge carriers with their different interpretation of knowledge represented according to "the same" schemes with the distorting effect between different representations.

For example, in spite of all seemed strangeness to ordinary thinking, at cognitive mapping the vagueness of the concept of the influence weight as well as ignoring the fact that in mathematical sense weights in a map characterize not only separate influences, but also proportions of different influences onto one factor, become significant risk factors for final result validity, along with known facts of cognitive biases in weight estimation. In some more detail the means and mechanisms of distortions are analyzed in (Abramova et al., 2009).

In terms of the model of knowledge transformations, the question about risks is whether it is possible to assume that cognitive means which are subconsciously used by a person when comprehending (primarily reflecting) a problem situation and subsequently formalizing the initial representations about it are identical with the formalized models of knowledge about a situation. (In fig.1 this question is marked as “”?3"). More exactly, obviously assuming the distinctions in above cognitive means, the question is, whether it is possible to consider the distorting effect brought by these distinctions, insignificant or, at least, acceptable.

According to the model considered, the distorting effect at change of cognitive knowledge representation means for "the same" knowledge is accepted as the basic mechanism of risk, so we can speak about cognitive risks in the course of transformations.

Note that formal transformation generating new knowledge about a problem situation from the entered knowledge (double-end arrow in fig.1) also could be considered as the risk factor for final result validity. However, today the theorists of formal science believe that formal methods are verifiable so it is not worthwhile to treat such situation as typical one. In section 3.2 we show the example disproving this belief with a distorting effect mechanism.

Stemming from the presented simplified model of cognitive knowledge transformations at decision-making in ill-structured problem situations, the analysis of human-induced risks for a specific technology and its decision-support system should include two stages of analysis: at the "macro-level" and at the "micro-level". At the macro-level the technological approach is required where the process of the new knowledge generation is described as the structured (according to the technology) collective activity. This activity participants and knowledge carriers are people and "formal experts"- computers, and both act according to the roles prescribed and tasks to be fulfilled. At the micro-level, the analysis of effects of psychological and other factors and mechanisms in the described process which can result in significant risks for final result validity should be carried out.

Our practice shows that this general methodology in whole is applicable to the cognitivemap-based methods, though further researches aimed at systematization of risk factors and their practical significance are required. 


\subsection{Model of cognitive control at formalization. Two kinds of risk factors}

Let us consider in brief the further development of the above ideas in (Abramova, 2002) concerning the stage of formalization. Fig. 2 visualizes the model from (Abramova, 2006) which treats the change of subject's cognitive means of knowledge representation at the stage of formalization as action of the imposed forms of thinking and interprets such action as the cognitive control. In the above model of cognitive transformations the subject is a decision-maker.

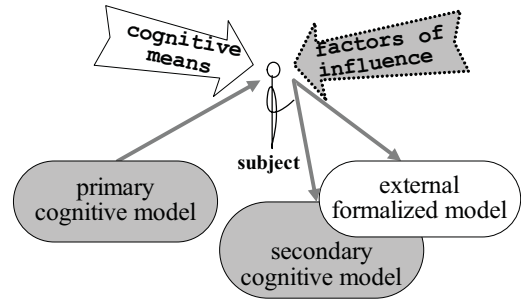

Fig. 2. Formalization of the subject's representations about a situation under cognitive control

The concept control is used in the sense accepted in the control science as a purposeful influence on the object of control; here a decision-maker turns to be the object of control. The purpose is formalization of primary knowledge about a problem situation in order to apply a formal method for new knowledge generation. Cognitive control means that control acts upon subjective cognitive means of the person interpreting a situation; first of all, these are his (or her) general concepts and knowledge representation schemes in terms of which the formalization of the person's internal representation of the situation occurs.

The internal representation of a situation is considered as the cognitive model of this situation. It is a model relative to a situation as well as any reflection in the mind of the person, and it is cognitive one as been formed with cognitive means. In the process in fig.1 cognitive control is carried out with imposing the prescribed cognitive means upon the expert at the formalization stage (i.e. expert knowledge elicitation), starting with means of structuring a situation: general concepts and theoretical models as schemes to formally represent knowledge about a situation. The example of such general concepts for cognitivemap-based formalization is given below.

In comparison with the above model of knowledge transformations (Fig. 1) the given model has one essential refinement. It is not only the external formalized model (the input information for a computer and/or the document if stipulated) that is treated as a result of the initial cognitive model formalization, but also the change in the state of an internal model of a situation leading to a secondary internal cognitive model. Such a concretization is essential in the design of computer support to formalization processes with taking account of distorting effects and planning protection against risks.

According to the principle of distorting effect, it is assumed that in generally all three models (fig.2) only more or less agree with each other. (In a specific case the effect can be unessential in the context of a solved problem.) Here it is possible to see deep analogy to the known relativity hypothesis of Sapir-Whorf telling that the picture of the world depends on a natural language, with the difference that the language means for structuring analyzed situations are over imposed onto natural ones.

The problem of the form and extent of discrepancy of the new situation models from the initial one is decisive for an adequate solution of practical problems. It is easy to admit that 
in a specific situation the result can depend not only on the means of formalization acting upon the subject, but also from himself and others' influences, besides imposed formalization means. Theoretical considerations, the analysis of practice and known facts of essential negative distorting effect have led to necessity to consider psychological factors of influence on the result and its correspondence to acceptability norms for practical problems under solution. In this context the major pragmatic problem consists in how to structure diversity of known factors with the purpose to identify relevant sources of risk factors and search for protecting means. To cope with arising questions, we consider two kinds of risk factors (Abramova, 2006).

The risk factors psychologically influencing validity of expert (or subject-formal) methods during their application by experts and analysts are referred to as first kind factors, or factors of direct action. Such factors can either objectively promote invalidity of results, or raise subjective confidence of experts of objective validity of the method application results. One can tell that they represent themselves as factors of belief. Conductors of these factors influence are experts and analysts; just they appear in conditions which may lead, eventually, to insufficiently valid (in the objective ration) results.

Second kind risk factors or factors of indirect action psychologically influence upon validity of subject-formal methods during their creation and substantiation. Conductors of influence of such factors are creators of methods, scientists and experts producing standards who, in turn, are subject to influence of scientific norms, paradigms, etc. At presence of second kind factors at the stage of formalization of primary representations first kind factors can be induced by action of second kind factors on experts and analysts.

In some cases the imposed forms and thinking stereotypes are not identified obviously so pertinently to speak about influence of ambient intelligence and myths. The interaction of cognitive means of knowledge formalization and other factors of influence which lead to cognitive biases are presented in fig. 3 .

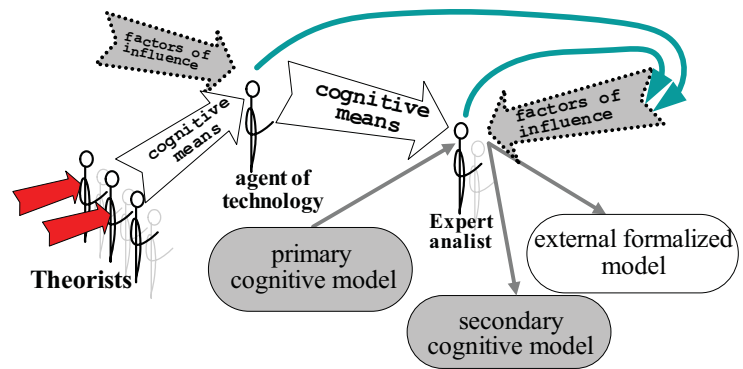

Fig. 3. Interaction of various factors on results of formalisation

As an example, one type of risk sources can be given, those acting regularly at the formalization stage, in particular, for cognitive-map-based methods. These are "two-faced" concepts (Abramova et al., 1999) that are usually entered as the interface concepts for elicitation of knowledge from experts and analysts (arrows 3,4 in fig.1). Such a concept has two "faces" that is two meanings turned to different people: a practical meaning for carriers of knowledge about problem situations and scientific one for carriers of scientific knowledge as well as information technology designers who try to convert more or less strict and sometimes complex scientific concepts into the "intuitively clear" language of vague natural concepts. 
A widely known example of such a concept which can create the significant distorting effect at the stage of formalization, is concept "linguistic variable" with verbal values represented in the computer on the numerical scale. This standard transformation (arrows 5, in fig.1) is put into a number of cognitive-map-based methods. It adds equality of distances between adjacent values to the ordering relation of verbal values, which the person means in his estimations (considerable distortion of initial meaning). With such formalization which does not take into account the specificity of subjective (personal) scales in specific situations, it may appear, for example, that a "low" (below average) man or house will be twice lower than "enough high" (above an average) one.

The transformation of scales is based on intuitive belief that "it's all the same" (identity 1 in fig.1), fixed in the scientific stereotype which makes this transformation a professional norm. The attempts to uncover the roots of this stereotype show that theorists and technology developers form in their mind some or other universal linguistic scale that does not depend on the context and the person. Such an ordinal scale admits formal transformations into the proportional numerical scale. Nevertheless it is intuitively clear from examples as well as experimentally proved that this induces essential distortions of expert knowledge. This idea of the way the decision-making personnel think when estimates weights, supported with the stereotype, turns to be the second kind risk factor. This second kind factor operates quite often with distorting the expert knowledge unnoticeably for its carriers (experts).

As the example shows, one of ways for revealing of second kind risk factors is checking the reasoning of knowledge transformations in transitions between formal knowledge of the computer and knowledge of people (Abramova, 2007; Abramova et al., 1999).

\section{Recently discovered risk factors due to the human-factor in cognitive-map-based subject-formal methods}

The proposed models and approaches for analysis, explanation, and forecasting of risks allow to systematize to some extent risks due to the human factor which, ultimately, can lower validity of subject-formal methods on the basis of cognitive maps with regard to: the place in the process of cognitive transformations of knowledge; the place in method life cycle; the human roles in this cycle. However, it is already clear, that even the set of risk factors discovered by now, makes a rather difficult cause and effect structure ${ }^{14}$.

To date our researches give considerable attention to two recently discovered problems (Abramova \& Kovriga, 2008; Abramova et al., 2009) which are caused by second kind risk factors. The first one is the problem of false transitivity of causal influences; and the second one is the problem of vagueness of semantics of general models and modelling languages when solving practical tasks by means of formal cognitive maps. The problem of false transitivity and the risks related are considered in section 5 together with its possible solutions. The problem of semantics vagueness is considered in the given section with emphasis on the analysis of its mechanisms and their causal relations.

The object of analysis carried out is modeling of situations by means of dynamic cognitive maps in the spirit of Roberts. Its results are briefly presented on a Fig. 4 in the form of illformalized cognitive map of risk factors relations, where all causal influences have identical content "increases" risk.

${ }^{14}$ In (Abramova et al., 2009) we have tried to present knowledge on some known risk. 
Let us define some concepts more concretely. General and concrete situation models appear in definitions of factors in the map on fig. 4.

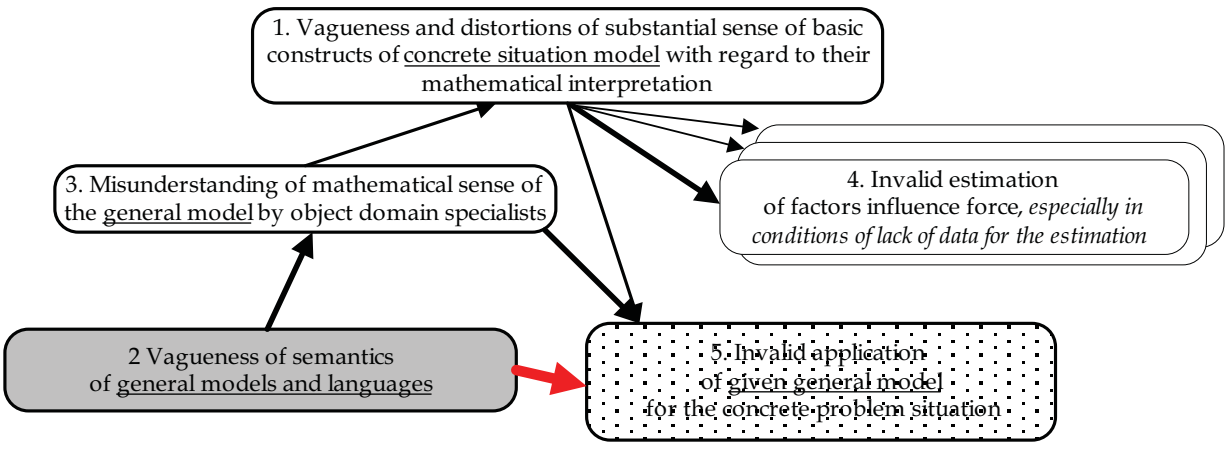

Fig. 4. Analysis of influence of models and languages semantics vagueness on the risks of invalid solution of problems by means of dynamic maps in Roberts' spirit

According to what has been said in section 2, it is supposed that general models for different type of maps form schemes of representation of knowledge about concrete situations at the stage of formalization, or in other words, languages for representation of such knowledge ${ }^{15}$. Concrete model of a situation is a result of formalization which is entered into the computer. In languages of situations modeling on the base of formal dynamic maps in the spirit of Roberts elementary constructs in situation description are factors, direct causal relations between them and weights of influences attributed to relations, which in aggregate form a cognitive map, in the combination with initial conditions which are attributed to factors.

Semantics of the certain modeling language is defined by basic constructs which, besides the named elementary constructs, include "node": the factor with all incoming direct influences together with the function of aggregation of (direct) these influences.

When analyzing the influences of the risk factor 2 we mean that the vagueness in all factors of the risk map (Fig. 4) may appear in the form of ambiguity of understanding and $\backslash$ or derived results between different people or even one person. Influences of the factor 2 can go through the chain $2 \rightarrow 3 \rightarrow 1$ to 4 and to other risk factors concerning different constructs defining semantics, which are not shown on Fig. 4.

Let us track, for example, the origination of risk through the typical chain $2 \rightarrow 3 \rightarrow 1 \rightarrow 4$. The intuitive metaphorical concept "influence weight", or its analogue "influence strength" which is used in all investigated type of maps in the spirit of Roberts, is vague in substantial sense: the expert is free to chose any of heuristic techniques for weighting particular influences when the valuation scale is given.

The mathematical sense of weight is specified by influences aggregation function, accepted in a certain theoretical model. In Roberts's base function (1) and its known modifications the weight of influences in aggregate reflect the proportions of separate influences. It means that

${ }_{15}$ In more strict terms of formal languages theory, the general model, as a rule, defines language semantics, and the language implements this model detailing semantics and syntax. 
estimation of weights is expedient within the context of other influences. However due to the vagueness of representation of weight semantics to experts - language users they may not know or not realize or underestimate the mathematical sense of weight, and it activates the chain $1 \rightarrow \longrightarrow 4$. Some available heuristic explanations of estimation of weights for real maps show that the specific character of mathematical sense of weights is ignored. Moreover, the known modern technologies of decision support on the base of formal dynamic maps in the spirit of Roberts make no provision for consideration of any context.

One more kind of risk, presented by the factor 5 , is activated in conditions when decisions on applicability of given general model of cognitive maps and associated subject-formal method should be made. This decision should be made on the basis of knowledge of the problem situation specialists a priori, i.e. prior to the formalization stage, but it can be changed in the course of formalization if irrelevances are revealed.

However in the presence of factor 1, there is a risk that invalidity of the given model and the problem solving method with regard to a concrete situation will remain unnoticed nor a priori (the chain of influences $2 \rightarrow 3 \rightarrow 5$ ), neither during formalization (the chain of influences $2 \rightarrow 3 \rightarrow 1 \rightarrow 5$ ). Some examples of such kind, when the one should refuse from application of the given model if there are no valid means to describe the situation by the given language, are presented in section 5 .

Let us consider another, more nontrivial example of results of influence of semantics vagueness of models and languages on risks of invalid problem solving in some known information technologies which realize modeling of cognitive maps in the spirit of Roberts. In this case the risk consisted in inadequate application of the general model to a concrete modelled situation (factor 5 on Fig. 4).

The vagueness of modeling language semantics in this case appears in the latent ambiguity of the concept initial condition which is non-terminal concept of this language. The mathematical concept "initial condition for the factor $x$ " in the meaning that is usually used for dynamic maps in the spirit of Roberts, is the number that expresses remainder $\Delta x(0)$ (according to the formula 1or similar formulae). From the point of view of dynamics, this concept has, at least two considerably different by kinds of the factor dynamics meanings (, according to the two graphically presented on Fig. 5).

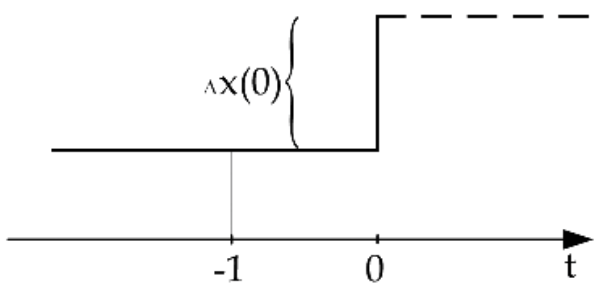

a) "Step"

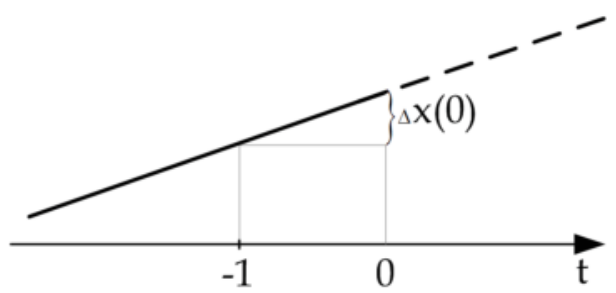

b) "Tendency"

Fig. 5. Two typical kinds of dynamics of cognitive map factors

The first one (Fig. 5a) is named step, or, in Roberts's terms, impulse which occurs at the moment of time modeling starts $t=0$. The number $\Delta x(0)$ expresses the step size. The second meaning matches in substantial sense with interpretation "tendency" and characterizes the dynamics when the speed of change of the variable remains constant, at least, on the average during some time, including the interval $(-1,0)$, and, probably, will remain constant, if there would be no reasons for its change (Fig. 5b). The number $\Delta x(0)$ expresses this speed. 
The first meaning corresponds to the verbal templates expressing initial conditions of type "factor $\mathrm{x}$ has strongly (or is weakly, moderately, ...) increased (or decreased)", and the second meaning - to the templates of type "factor $\mathrm{x}$ is strongly (or weakly, moderately, ...) increasing (or decreasing)".

Essentially, the choice of one of these two semantic meanings for representation of observable dynamics of factors leads to different models of behaviour (indiscernible within the frameworks of existing vagueness) and to different applied statements of modeling problems. Thus, if one of the models is applicable to the concrete situation, for applied reasons, the second one is not.

In the aforementioned technologies, where the considered risk was actualized, the widely known modeling technique coming from Roberts is applied [Section 2]. This technique allows to define the cumulative effect from simultaneous steps in all or in the part of factors, in the course of the transient process (if the one is stabilized). However for representation of initial conditions and results the users are being actually offered another model - in the form of verbal templates of the second kind or even with obvious implementation of the term tendency into the dialogue. Thus the technology actually realizes the following chain of transformations: initial tendencies $\Rightarrow$ initial steps $\rightarrow$ steps processing $\rightarrow$ cumulative change of values $\Rightarrow$ new tendencies, including the change of substantial sense of the initial data on dynamics of factors of a map and the results of modeling, while exchanging data between the person and the computer. Thereby the method of modelling of results of impulse (spasmodic) change in dynamics of a situation is applied to situations of self-development, where the steps, but not tendencies take place.

The nontriviality of the situation considered is that the risk was actualized only via the factors of the second kind, escaping the experts and analysts (direct influence $2 \rightarrow 5$ on Fig. 4).

\section{Some principles of cognitive mapping validity increase}

For cognitive mapping validity increase we put forward a number of principles. The principles include the following.

a. Accumulating experience of typical errors and risk factors.

b. Early detection and blocking of risk sources and semantic errors of formalization not putting obstacles in the way of further application of a chosen type of a cognitive map and a map-based method. Protection and blocking of risk sources is provided by adjustment of formalized knowledge with control of risks.

c. The chosen type of cognitive map applicability walkthrough for particular situation, including a priori and a posteriori control.

The proposed principles are directed to development of the following methods and supporting informational technologies: cognitive mapping by expert and verification of the cognitive maps.

Analysis of risks due to the human factor discovered by now (see Section 4) indicates advisability of statement and research of verification problem for modelling on the base of cognitive maps (see Section 5.1).

The questions of formalization validity criteria for realization of aforementioned principles also are still open. At that, the criteria applicable at early stages, i.e. until formal processing of formal model of experts' initial knowledge, are of special interest. 
We propose a number of validity criteria for formalization of expert knowledge by means of cognitive maps intended for more effective detection of direct errors and risks for validity of formalization results (see Section 5.2).

The criteria are local, i.e. these are applied to single constructions of a cognitive map of a situation. The locality property simplifies and systematizes the process of their application by both experts compiling cognitive maps and verifiers carrying out control of formalization correctness.

\subsection{Problems and principles to verification of cognitive maps and models}

Taking into account the risks discovered (Section 4), we distinguish two verification problems:

a. Verification of well-known formal cognitive map models.

b. Verification of cognitive-map-based situation models while solving real-world problems.

We develop a verification approach relying on experience of practical software verification. At that, this approach has a specific feature: verification is considered, in the first place, as a human activity, and formal methods are the means supporting such activity. The validity criteria system in general case is predetermined only partly and can be extended by experts in the course of particular verification ${ }^{16}$. As it is proved by practice and experiments, just this approach turns to be effective for modelling of problem situations on the base of cognitive maps.

Verification of general cognitive-map models. The principle of verification of modelling language semantics for ill-structured situations on the base of cognitive maps according to criterion of clarity of general theoretical models was proposed within the framework of verification problem for general theoretical models on the base of cognitive maps (Abramova, 2007). This is conditioned by selection of vagueness of models and languages for formalization of initial expert knowledge as a practically significant risk factor. This vagueness results in the ambiguity of interpretation of the general model. This is also confirmed experimentally. We propose a technique for verification based on developed earlier approach to formalization of description of different theoretical models of functional scheme types (Abramova, 1993). The technique has passed partial examination for some types of cognitive maps based models. As a result, we have discovered vagueness of semantics of several well-known theoretical models of cognitive maps and have carried out correction with "splitting" these models to alternative variants with significant difference in their properties and correct problem statements for forecast of dynamics of analyzed objects and situations.

Verification of particular cognitive maps that are built while solving real-world problems of modelling and control is aimed at:

- recognition of risky local fragments of formal model under construction and direct semantic errors of formalization;

- $\quad$ early control of applicability of the chosen type of general model of cognitive maps via correction of a priori estimations (assumptions) of its applicability to a particular situation in course of formalization.

\footnotetext{
16 in psychological terms, cognitive control of experts works
} 
Further research is directed to development and empirical testing of methods for verification of general and particular cognitive maps taking into account known (discovered before) factors of risk due to the human factor for validity, to their application to informational (cognitive) technologies of decision making support.

\subsection{Some criteria of cognitive maps validity}

To reduce the risks due to the human factor, we propose and ground a number of heuristic criteria of formalization validity that can be applied for early detection of direct semantic errors and risks of formalization. These criteria can be applied both at the stage of model construction and stage of verification.

All criteria that we obtained form the following two groups:

- Criteria that can be applied to individual formalization.

- Criteria of collective knowledge conformity.

These criteria were formulated mainly as applied to the cognitive maps in the spirit Roberts (see Section 2.1). However, degree of generality of the proposed criteria allows to expect applicability to other cognitive map types.

Here we consider some criteria applicable to individual formalization.

In view of the hypothesis about the negative distortion effect (see Section 3), which was proved while analyzing the practice of solving problems by means of cognitive maps, the general adequacy criterion for interpretation of substantial knowledge about a problem situation to the language of the mathematical model and backward is in the foreground of risk examination.

This criterion is related to:

- interpretation of intermediate substantial model of initial knowledge formulated by an individual while constructing a cognitive map of a particular problem situation to language of mathematical model being formally processed;

- backward interpretation of the mathematical model to a verbal form providing adequate understanding of the map.

At that, the backward interpretation implies understanding on substantial level of those mathematical properties that are attributed to formalized knowledge and thereby to modelled reality. Understanding of these properties creates the base for examination of applicability of the chosen model type to formalization.

Distortions are possible while constructing both a particular cognitive map (first kind risk factors) and templates of verbal interpretation of the map elements by theorists and developers of corresponding informational technologies (second kind risk factors).

The general criterion of interpretation adequacy is related to the group of particular criteria of cognitive clarity and the criterion of (balanced) completeness of influences to a factor considered in more details in Sections 5.2.1 and 5.2.2, correspondingly.

The criteria of extensions proportionality of factor's concept are formulated as a result of detection of causal influence false transitivity risk in cognitive maps. These criteria are considered in Section 5.2.2.

To demonstrate the discovered risks we use the example of the well-known cognitive map as well as fragments of real cognitive maps for solving particular real-world forecast and control problems.

\subsubsection{Criterion of mathematical model cognitive clarity}

The concept of cognitive clarity of some information, messages, descriptions, etc., characterizes easiness of intuitive understanding. The lack of cognitive clarity appears when 
a person has much ado, hesitates trying to understand what has been said or written ${ }^{17}$. This can appear in observed deceleration of understanding process.

With the purpose of adaptation of mathematical language of a model and increasing its cognitive clarity for specialists in a problem area, developers of informational technologies create verbal templates of interpretation of relations in a cognitive map to natural language, in other words, interpretation templates. The relation interpretation template is the verbal formulation of content of arbitrary relation in a map that is made specific by substitution of free variables for particular names of factors connected by direct influence. The templates of such kind are introduced, for example, in (Maruyama, 1963; Roberts, 1976; Abramova et al., 2008). As a simple example, let us consider a relation interpretation template expressing positive influence of a factor to another one: increase (decrease) of <name of factor $1>$, all other things being equal, causes increase (decrease) of <name of factor $2>$.

Substituting particular factor names for free variables in this template we define concretely the relation between these factors while constructing a cognitive map of a situation. While substituting particular factor names, for example, "amount of garbage per area" and "bacteria per area" we obtain the following: increase (decrease) of amount of garbage per area, all other things being equal, causes increase (decrease) of bacteria per area.

In this connection, we can speak of the cognitive clarity from two points of view. On the one hand, stereotyped nature of means of understanding really assists cognitive clarity of onetype constructions. But, on the other hand, with regard to published templates of map relations understanding, nowadays one can see a tendency to simplification of the templates to the prejudice of adequacy of mathematical sense of constructions. Thereby the cognitive clarity of a mathematical model is decreased for the problem area specialists who understand the mathematical sense via verbal templates. An example of application of simplified template creating the risk of inadequate application of the chosen mathematical model to the particular problem situation is considered further by the example of M. Maryuama's cognitive map (Maruyama, 1963).

The cognitive clarity of a situation mathematical model must be provided:

- at the stage of construction of general formal models of cognitive maps or while improving their semantics for user via templates;

- $\quad$ at the stage of construction of particular situation models on the base of cognitive maps. The cognitive clarity criteria include a group of local particular criteria relating to separate constructions of a map such as a factor, relation, factor with the set of all factors influencing it. Let us consider some of them.

Criteria of normality of factor concept form. In cognitive maps, the factor concepts are twofaced, i.e. they bear double sense (Abramova, 2007). On the one hand this is a factor in a substantial sense. On the other hand, this is a variable possessing values at a definite measuring scale or estimation scale with linearly ordered set of values.

Vagueness and distortions of the substantial sense of model constructions with regard to their mathematical interpretation cause the risk of unreliable estimation of factor influence

17 We use the term "cognitive clarity" instead of more habitual "clarity" to emphasize that estimations of these property (within a context of risk problem) have to be made not on the base of a "common sense" of developers of formal models, methods and corresponding informational technologies, but on the base of knoledge of cognitive science and empirical data. 
forces, especially for the lack of data for such estimation. One of the risk factors is incomplete understanding of mathematical sense of constructions by a problem area specialist. Such incomplete understanding, in turn, creates the risk of inadequate application of the chosen general formal model to a particular problem situation.

Based on aforesaid, we propose a criteria of normality of factor concept form $\mathrm{Kc}(\mathrm{p})$. It is assumed that the factor concept $\mathrm{p}$ is named in normal form if it can be naturally interpreted (understood) as both a factor in substantial sense and a variable taking on values at a definite measuring or estimating scale. If $\mathrm{K}^{\mathrm{c}}(\mathrm{p})$ is met for a factor that is understood in a substantial sense, then the clarity of mathematical model of this factor in the form of a variable of a definite type is provided.

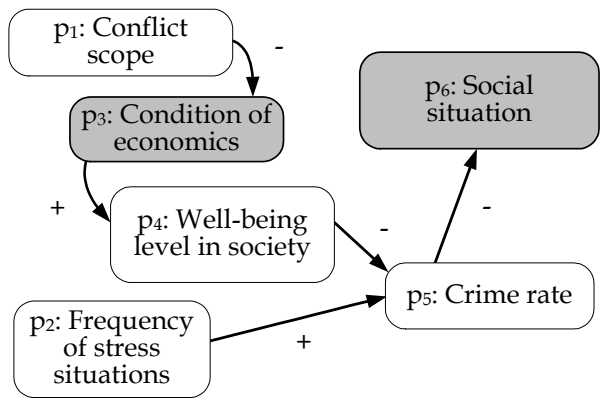

a) Initial map

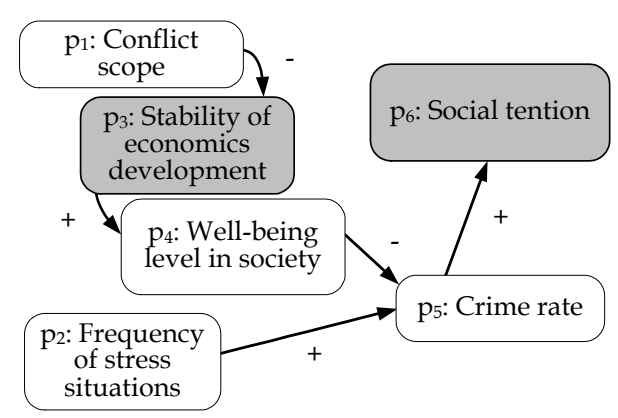

b) Improvement

Fig. 6.1. Fragment of the cognitive map of conflict situation and its improvement

Figure 6.1a contains a fragment of the cognitive map of a conflict situation that was constructed based on the results of analysis of experts' representations of development of conflict in former Yugoslavia.

According to the criterion $\mathrm{K}^{\mathrm{c}}(\mathrm{p})$, the concepts of factors $\mathrm{p}_{1}, \mathrm{p}_{2}, \mathrm{p}_{4}, \mathrm{p}_{5}$ are named in normal form. These factors can be naturally considered as the variables taking on values either from numerical scale (if they are measurable) or from estimating scale with verbal values such as "high", "low", etc.

In the view of linguistics, normality appears in natural use of named factor concepts in verbal context such as "more - less", "increase - decrease", "to increase - to decrease", etc. The concepts of factors $\mathrm{p}_{3}$ "condition of economics" and $\mathrm{p}_{6}$ "social situation" are the exceptions. Need to use these concepts in such verbal contexts can result in difficulties and one may need certain intellectual effort to find at least content-similar contexts such as "worse - better", etc. This fact shows lack of cognitive clarity of the concept as a variable of the required type.

Correction of a concept in case of its mismatch to the criterion $\mathrm{Kc}^{\mathrm{c}}(\mathrm{p})$ can be made by solely linguistic means without taking into consideration expert knowledge about the a matter of a concept .considered. For example, the concept "condition of economics" can be substituted for "quality of economics condition". However, more valid modelling can be achieved taking into account expert knowledge, at that not only with respect to a matter of concept, but its causal relations with other factors. Then in the considered example one can choose the concept "stability of economics development" instead of "condition of economics" and "social tension" instead of "social situation". Such improvement required change of influence sign between the factors $\mathrm{p}_{5}$ and $\mathrm{p}_{6}^{\prime}$ (see fig. 6.1b). 
This example shows that mismatch between the concept and the criterion $K^{c}(p)$ can be interpreted as insufficient clearness of substantial sense of the factor with respect to the required mathematical sense. Vagueness is the risk factor for the model validity that is discovered as a need in improvement and corrections caused by examination of conformity with the criterion $\mathrm{Kc}^{\mathrm{c}}(\mathrm{p})$. This becomes most evident while converting relations including incorrect concepts to mathematical language.

Criterion of intelligibility of mathematical sense of map construct by verbal template. Intelligibility of mathematical sense of a map construction (in particular, a relation) by verbal template means that to read a certain mathematical property of a construction, for a problem area specialist is sufficient to understand its expression obtained by application of a verbal template for interpretation of corresponding property into a natural language.

More exactly, the sense of the criterion of comprehensibility of relation by template $\mathrm{K}^{\mathrm{b}}\left(\mathrm{b}_{0}, \mathrm{Sh}\right)$ applicable to any relation $b_{0}$ in a cognitive map consists in the following: the mathematical property assigned to the relation $b_{0}$ by the template $S h$ in accordance with semantics of the general model must be clear for an expert such that adequacy of this property or, at least, its acceptability for expression of the causal relation represented by $b_{0}$ from substantial point of view can be estimated.

Criteria of the type $\mathrm{K}^{\mathrm{b}}\left(\mathrm{b}_{0}, \mathrm{Sh}\right)$ with a given templates serve as an auxiliary mean of verification of modelling validity of observable (or assumed) causal relations of factors. Neglecting of examination in accordance with such criteria results in the risk of invalid application of the chosen formal model to a particular situation that can cause invalidity of final results. This is in full conforms the results of research described in (Richardson, 1997).

The distortion of substantial sense of the separate influence, caused by intuitive simplification of the transformation template, creates the risk of inadequate application of the chosen mathematical model to a specific problem situation. This risk may come to effect, for example, in the invalidity of the model application to the specified situation.

The given risk is that of the first kind as far as the distortions are made by people who derive cognitive maps. However, if (1) the misunderstanding of the mathematical sense of the influences is caused by the fact that the designers of decision support technology do not provide a user with, at least, the information on the formal sense of the basic model constructs; or (2) the risky template is included in the definition of semantics of the model which is the basis of the derived cognitive map; the one can reasonably talk of the semantic vagueness as of the risk factor of the second kind that causes distortions.

The detailed description of one of mechanisms of origin of erroneous recommendations given to decision-making personnel that are caused due to ignoring the risk factors at the stage of formalization of initial representations about a problem situation with Maryuama's map is presented in (Abramova \& Kovriga, 2008).

\subsubsection{The risk of causal influences false transitivity and criteria of factor's concept extensions proportionality}

The one can say about violation of transitivity of causal influences if when $\mathrm{A} \rightarrow \mathrm{B}$ (" $\mathrm{A}$ causes $\left.\mathrm{B}^{\prime \prime}\right)$ and $\mathrm{B} \rightarrow \mathrm{C}, \neg(\mathrm{A} \rightarrow \mathrm{C})$ actually takes place instead of $\mathrm{A} \rightarrow \mathrm{C}$ what is expected according to the transitivity rule. In other words, $\mathrm{C}$ does not essentially depend on $\mathrm{A}$, so $\mathrm{A}$ is not an indirect cause of $C$.

At the same time the causal influences transitivity principle is generally accepted as universal. Accepted as an axiom when modelling situations by means of cognitive maps and other formal methods, it provides an automatic inference of indirect influences. 
The risk of violation of causal influences transitivity, on the one hand, has been supposed by authors as a hypothesis coming from theoretical considerations (by analogy to known violation of transitivity of paired preferences "despite obviousness"), and, on the other hand, - violations have been actually found in practice when constructing and applying dynamic maps in order to solve ill-structured situations control problems.

The fragment of the real cognitive map with discovered more complex case of false transitivity through long chains of influences between factors is presented on Fig. 8. The map has been created to analyze the problems related to narcobusiness and drugs use in country " $N$ " which has transit narcotraffic on its territory.

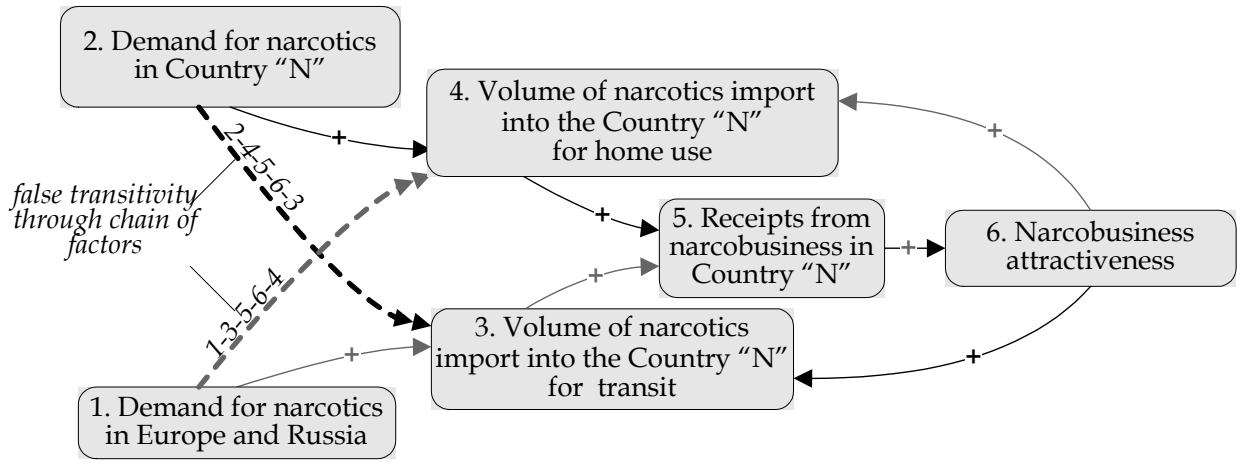

Fig. 8. Fragment of real-life cognitive map of narcosituation with false transitivity

Presented fragment of the map is completed with two indirect influences $2 \stackrel{+}{\longrightarrow} 3$ and $1 \stackrel{+}{\longrightarrow}$ which "are logically deduced" from the chains of direct influences $2 \stackrel{+}{\longrightarrow} 4 \stackrel{+}{\longrightarrow} 5 \stackrel{+}{\longrightarrow} 6 \stackrel{+}{\longrightarrow} 3$ and $1 \stackrel{+}{\longrightarrow} 3 \stackrel{+}{\longrightarrow} 5 \stackrel{+}{\longrightarrow} 6 \stackrel{+}{\longrightarrow} 4$ accordingly through the transitivity axiom. (Indirect influences are shown by a dotted line).

According to the verbal template of interpretation of influences for Roberts' linear dynamic maps (Roberts, 1976), the mentioned indirect influences $2 \stackrel{+}{\longrightarrow} 3$ and $1 \stackrel{+}{\longrightarrow} 4$ mean the following: an increase (decrease) in Demand for narcotics in Country " $\mathrm{N}$ " causes an increase (decrease) in "Volume of narcotics import into the Country "N" for transit"; an increase (decrease) in "Demand for narcotics in Europe and Russia" causes an increase (decrease) in "Volume of narcotics import into the Country "N" for home use".

However the direct estimation of presence of influences in pairs $2 \longrightarrow 3$ and $1 \longrightarrow 4$ for substantial considerations says that actually in each pair the factors are independent. Thereby in both cases false transitivity takes place.

In this case, in the chain 2-4-5-6-3 not any change in "Receipts from narcobusiness in Country "N" (5), and therefore in "Narcobusiness attractiveness" (6) is caused by change in "Volume of narcotics import into the Country " $\mathrm{N}$ " for home use" (4), and in the following influence $6 \rightarrow 3$ a change in "Narcobusiness attractiveness" (6) not necessarily changes "Volume of narcotics import into the Country "N" for transit" (3). In the second chain $\stackrel{+}{\longrightarrow} 3 \stackrel{+}{\longrightarrow} 5 \stackrel{+}{\longrightarrow} 6 \stackrel{+}{\longrightarrow} 4$ the situation is similar.

Substantially it is possible to explain the false transitivity by the presence of risky (in logic sense) combinations of causal influences in the chain that generates it (transitivity). In this 
case, in the chain 2-4-5-6-3 not any change in "Receipts from narcobusiness in Country " $\mathrm{N}$ " (5), and therefore in "Narcobusiness attractiveness" (6) is caused by change in "Volume of narcotics import into the Country " $N$ " for home use" (4), and in the following influence $6 \rightarrow 3$ a change in "Narcobusiness attractiveness" (6) not necessarily changes "Volume of narcotics import into the Country "N" for transit" (3). Therefore the given chain of factors does not mean obligatory indirect influence on all chain, and more detailed analysis of influences is required. In the second chain $1 \stackrel{+}{\longrightarrow} 3 \stackrel{+}{\longrightarrow} 5 \stackrel{+}{\longrightarrow} 6 \stackrel{+}{\longrightarrow} 4$ the situation is similar.

From the applied point of view, significant aspects of a problem of false transitivity of causal influences when solving concrete practical problems, are the early recognition of situations modelled by means of cognitive map, that can become the reason of false inferences, and their diagnostics for decision-making on correction or even, if the one is impossible, to refusal from the technique chosen.

The analysis of fragments of other cognitive maps with false transitivity shows that their general feature is presence disproportionately general in extension of concepts of factors comparatively to other factors in the chain of direct influences. However the presence of such concepts not always leads to false inferences through transitivity, so we can talk only about risks that require further analysis.

Let us give the formalized definitions of criteria of factors concept' extension proportionality, offered in (Abramova \& Kovriga, 2008) to help experts to recognize such risky fragments.

In the definition we take into account the fact that has been found out in practice: the same (as a matter of fact) causal influence may be represented in a cognitive map by different concepts so that one may speak about different representations of the influence. Suppose we have factors $A, B_{1}$ (represented with the concepts of the same name), which are linked by direct causal influence $B_{1} \rightarrow A$, and let there exists (is found by an expert) factor $B_{2}$ such that replacement of representation of influence $B_{1} \rightarrow A$ with $B_{2} \rightarrow A$ does not change the influence substantially, and herewith

$$
v_{B_{1}} \supset v_{B_{2}}
$$

where $v_{B_{i}}, i=1,2$ - the extension of the corresponding concept, and the relation between extensions is treated as usual set-theoretical inclusion or, that is the same, verbally: " $B_{1}$ has bigger extension than $B_{2}$ ", “ $B_{1}$ is more general than $B_{2}$ ".

Then factor $B_{2}$ is more proportional in its concept extension then $B_{1}$ as the source in the direct influence on $A$, and factor $B_{1}$ is extensionally excessive in this influence on $A$. In other words the concept of factors-sources (causes) is too general comparatively to the factors-receivers (effects), as far as the expert has found less general concept to represent the known influence.

The proposed expert criterion of extensional proportionality $\mathrm{K}^{\mathrm{S}}(\mathrm{B}, \mathrm{A})$ is applicable to any pair of factors of a cognitive map, connected by direct influence.

The criterion of extensional proportionality for the influence receiver $K^{D}(A, B)$ is formulated and applied similarly, though in case of many influences on one factor it is less informative for risk detection and error correction.

Using the presented criteria an expert can easily understand in the example considered (fig. 7 ), that, for example, the concept (5), "Receipts from narcobusiness in Country " $\mathrm{N}$ ", 
designating the influence receiver, is excessive in its extension comparatively to the source of influence (4), "Volume of narcotics import into the Country " $\mathrm{N}$ " for home use" as far as the influence actually concerns only "Receipts from narcobusiness in Country " $\mathrm{N}$ " coming from home use". Similarly excessive in their extensions are the concept of the factor (5) as the influence receiver comparatively to (3) and concept of the factor (6) as an influence source comparatively to (3) and (4).

Let us underline that when allocating disproportionally general concepts on a map using the proposed criteria a question on the ways it can be corrected to except false inference and on the possibility of such correction still remains open.

In rather simple cases with short chains of false transitivity we managed to avoid it by correcting the initial cognitive map. (Abramova \& Kovriga, 2008) gives a description of such correction by splitting of the too general concept. The correction considerably changes the results of dynamics of the considered situation and control of its development in order to increase the volume of gas production in the country.) The correction essence is that the way of representation of causal influences known to experts has been changed.

However the considered fragment fig. 7 and other similar examples uncover the following: in internal causal cognitive maps of complex situations experts can have concepts of different degree of generality and corresponding conclusions about the influences. Therefore in the general case the possibility of their representation in the form of "a singlelevel" formal map is doubtful. The further research of real maps and, probably, complication of applied formal theoretical models of cognitive maps is required.

Finishing the analysis of a problem of risks of causal influences false transitivity in cognitive maps, we will notice that, as shown in (Abramova \& Kovriga, 2008), these risks are caused by joint action of two risk factors, including

- disproportion of concepts generality, typical when conceptualizing complex and illstructured situations;

- the transitivity axiom, that is supported by belief in universality of causal influences transitivity principle.

The disproportion of concepts generality is a first kind risk factor, which in practice comes from both experts and analysts, and intermediaries in formalization; the transitivity axiom is an ineradicably risk factor of the second kind which, by default, is introduced in the end result at the stage of a map formal processing.

\section{Concluding remarks}

State-of-the-art in the field of both theory and practice related to formal cognitive maps shows two tendencies. On the one hand, experience of their practical applications is accumulated that testifies to their utility for problem solving for ill-structured situations and qualitative knowledge of situations. On the other hand, knowledge of human-induced risks is accumulated as well, this knowledge being not only theoretical one, but also in the form of examples of embodied risks in concrete maps and map-based models.

According to principles of protection against risks proposed in (Abramova, 2007), further research aimed to decrease of riskiness of cognitive-map-based methods should move in two directions: 
- development of protective methods for inclusion into human-dependent stages of information technologies supporting existing formal methods beginning from stages of formalization and up to verification of cognitive maps and map-based models;

- development of advanced methods taking account regular human factors (such as false transitivity of causal influences) through all stages of knowledge transformations.

Today the list of human-induced risks and direct errors typical for cognitive-map-based models and methods as well as the list of known criteria for early detection of such risks and errors are far from end; further empirical research into this problem is required (aimed at systematization of risk factors and their practical significance: both as to factors specific to formal cognitive maps and their separate types and general for different subject-formal decision support methods).

As the most significant challenge, following from discovered risk factors of regular action, the problem of false transitivity of causal influences should be treated as worthy of consideration both as the fundamental problem of correct expression of such influences, and as the technological problem of such risk detection with restriction on the analysis time. The following fact discovered in practice seems to be of fundamental importance in the context of this problem: the same (substantially) causal influence can be presented by means of various concepts on a cognitive map (Abramova \& Kovriga, 2008). Thereby the problem of false transitivity of causal influences in its fundamental aspect appears closely related to logic and linguistics, being the interdisciplinary problem.

At last, it is necessary to designate the problem of training of users of information technologies based on formal dynamic cognitive maps for modeling ill -structured situations. As researches (Schaffernicht, 2007) have shown, teaching the general concepts which characterize dynamics of processes ("behavior" in contrast to events) brings in difficulties and is connected with cognitive biases. This is also confirmed by our practice and the analysis presented herein. One of the ways to solve this problem, in our opinion, is to develop cognitive training simulators which will help users' model dynamics of complex situations for definite types of cognitive maps in the context of known problem situations.

\section{References}

Abramova, N.A. (2007). On the problem of risks due to the human factor in expert methods and information technologies. J. Control Sciences, No. 2, (Mar/Apr. 2007), (11-21), ISSN: 1819-3161.

Abramova, N.A. (2006). A subject of intellectual activity under cognitive control of ambient intelligence, Proceedings of 9th IFAC Symposium on Automated Systems Based on Human Skills and Knowledge, pp. 73-78, ISBN:, Nancy, France, 2006. http://www.ifac-papersonline.net/Detailed/38844.html

Abramova, N.A. (2002). On forming the interface concepts of computer technologies and on psychological correctness, Proc. of 2th Intern. Conf. "Cognitive analysis and Situations evolution Control, vol. 2, pp.26-35, ISBN: 5-201-149646-4, Moscow. Institute of Control Sciences. 2002.

Abramova, N.A. (1993) General approach to the analisys of external behavior of the plants , presented by functional diagramms, based on equvalent transformations, 
Automation and Remote Control, Vol. 2, No. 3, (June1993), (115-134), ISSN : 0005 2310

Abramova, N.A.; Kovriga S.V. \& Makarenko D.I. (2009). One Approach to Analysis of Risks due to Human Factors in Decision Support Systems for Ill-Structured Situations, Proceedings of Conference on Human System Interaction - HSI'2009, pp. 113-123, ISBN:1-4244-1543-8, Catania, Italy, May 21-23, 2009.

Abramova, N.A.; Avdeeva, Z.K. \& Kovriga, S. V. (2008) Cognitive Approach to Control in Ill-structured Situation and the Problem of Risks. In: Advances in Robotics, Automation and Control, J. Aramburo and A.R. Trevino (ed.)., (85-110), IN-TECH, ISBN : 978-953-7619-16-9, Viena, 2008. -

http:/ /intechweb.org/ book.php?id=39\& content=new\&sid=1.

Abramova, N.A. \& Kovriga, S.V. (2008). Cognitive Approach to Decition-maiking in IllStructured Situation Control and the Problem of Risks, Proceedings of Conference on Human System Interaction - HIS'2008, pp. 83-88, ISBN:1-4244-1543-8, Krakow, Poland, May 25-27, 2008.

Abramova, N.A.; Kovriga, S.V. et al. (1999) Seaching of Approach to Problem Solving. SYNTEG, ISBN: 5-89638-018-6, Moscow,1999.

Avdeeva, Z. \& Kovriga, S. (2008). Cognitive approach in simulation and control. Plenary papers, Milestone reports $\mathcal{E}$ Selected survey papers. 17th IFAC World Congress, pp.160167, ISBN: 978-3-902661-00-5, Seoul, Korea, July 2008. http://www.ifac-papersonline.net/Detailed/36001.html

Avdeeva, Z.; Kovriga, S. \& Makarenko, D. (2007). Cognitive approach to problem solving of social and economic object development. Proceedings of 4th International Conference on Informatics in Control, Automation and Robotics, pp. 432-435, Angers, France, may 2007.

Axelrod, R. (1976). The cognitive mapping approach to decision making. In: Structure of Decision. The Cognitive Maps of Political Elites, R. Axelrod (ed.), (3-18), ISBN: 069107578-6, Princeton University Press, Princeton.

Chaib-draa, B. (2002). Causal maps: theory, implementation, and practical applications in multiagent environments. J. IEEE Trans. on Knowledge and Data Engineering, Vol.14, №6, (november/december 2002), (1201-1217), ISSN: 1041-4347.

Bell , D.E.; Raiffa, H. \& Tversky, A. (ed). (1988). Decision Making: descriptive, normative and prescriptive interactions, (9-32), Cambridge University Press, Cambridge.

Bouzdine-Chameeva, T. (2006). An application of causal mapping technique ANCOM-2 in management studies, Proceedings on the 6th Global Conference on Business $\mathcal{E}$ Economics, pp. 11-21, ISBN: 0-9742114-6-x, USA, oktober 2006, Gutman Conference Center, USA.

Dörner, D. (1997). The Logic of Failure: recognizing and avoiding error in complex situations, Basic Books, ISBN: 978-0201479485, Massachusetts.

Eden, C.; Ackerman, F. \& Brown, I. (2005) The Practice of Making Strategy: Step by Step Guide, Stage, ISBN:076194494 X(pbk), London

Fedulov, A.S.(2005). Fuzzy relational cognitive maps. J. of Computer and Systems Sciences International, , Vol.44, No.1, (2005), (112-124), ISSN: 1064-2307 
Gorelova, G.V. \& Zaharova E.N.(2006) Structured Analysis of Complex Systems Cognitive Models. Proc. of VIth Intern. Conf. "Cognitive analysis and Situations evolution Control, vol. 2, pp.26-35, ISBN: 5-201-149646-4, Moscow. Institute of Control Sciences. 2006.

Heyer, R. J. (1999). Psychology of Intelligent Analysis. Central Intelligence Agency. ISBN: 1 929667-00-0

Hodgkinson, G.; Maule, A. \& Bown, N. (2004). Causal Cognitive Mapping in the Organizational Strategy Field: A Comparison of Alternative Elicitation Procedures, Organizational Research Methods, Vol. 7, No. 1, (Jan. 2004), (3-26), ISSN: 1094-4281, SAGE Publications

Kim, D. (2000). A simulation method of cognitive maps. Proceedings of 1st International Conference on Systems Thinking in Management., pp. 294-299, 2000, Geelong, Australia, Deakins University, Geelong.

Kremer, R. (1994). Concept mapping: informal to formal, Proceedings of the Second International Conference on Conceptual Structures, pp. 45-55, ISBN: 3-540-58328-9, College Park, Maryland, USA, Aug. 1994, Springer, Maryland.

Kulba, V.V.; Kononov, D.A. \& other. (2004). Methods for forming development scenario for socioeconomic systems, SYNTEG, ISBN, Moscow.

Kuznetsov, O.P.; Kilinich, A.A. \& Markovskii, A.V. (2006). Influence analysis in control of illstructured situations based on cognitive maps. In: Human factor in control sciences, $\mathrm{N}$. Abramova, D. Novikov, and K. Hinsberg (ed.), (311-344), URSS, ISBN: 5-484-003911, Moscow.

Larichev, O.; Moshkovich H. (1997). Verbal decision analysis for unstructured problems, Kluwer Academic Publishers, ISBN: , Boston.

Maximov, V. \& Kornoushenko, E. (2001). Analytical basics of construction the graph and computer models for complicated situations. Proceedings of the 10th IFAC Symposium on Information Control Problems in Manufacturing, pp.113-225, Vienna, Austria, September 2001.

Maruyama, M. (1963). The Second Cybernetics: Deviation-Amplifying Mutual Causal Processes. J. Amer. Sci., Vol. 5, No. 2, (June 1963) (164-179f), ISSN: 0003-0996, California.

Narayanan, V. K.\&Deborah, J. (2005) Causal mapping for research in information technologies, Idea groop, ISBN: 1-59140-396-0, Hershey, USA

Peña, A.; Sossa, H. \& Gutiérrez, A. (2007) Cognitive maps: an overview and their application for student modeling. J. Comp. y Sist., Vol.10, №3, (Jan./Mar. 2007), (230-250), ISSN: 1405-5546.

Richardson, G. P. (1997) Problems in causal loop diagrams revisited. System Dynamics Review, Vol. 13, No. 3, (Fall 1997): 247-252

Roberts, F. (1976). Discrete mathematical models with applications to social, biological and environmental problems. Prentice Hall, ISBN: ISBN-13: 978-0132141710, New Jersey.

Schaffernicht, M. (2007). Causality and diagrams for system dynamics. Proceedings of the 25th International Conference of the System Dynamics Society, pp. 24-49, ISBN: 978-09745329-8-1, Boston, USA, July 29 - August 2, 2007. 
Vesa, A. N. (2007). Application of Fuzzy Cognitive Maps to Business Planning Models. In : Theor. Adv. and Appl. of Fuzzy Logic, O. Castillo et al. (ed.), Vol. 42 , (119-127), Springer-Verlag, ISBN: 978-3-540-72433-9, Berlin/Heidelberg. 


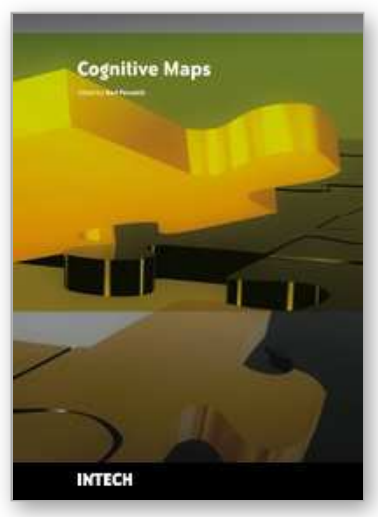

\author{
Cognitive Maps \\ Edited by Karl Perusich
}

ISBN 978-953-307-044-5

Hard cover, 140 pages

Publisher InTech

Published online 01, January, 2010

Published in print edition January, 2010

\title{
How to reference
}

In order to correctly reference this scholarly work, feel free to copy and paste the following:

Abramova N., Avdeeva Z., Kovriga S. and Makarenko D. (2010). Subject-formal Methods Based on Cognitive Maps and the Problem of Risk Due to the Human Factor, Cognitive Maps, Karl Perusich (Ed.), ISBN: 978-953307-044-5, InTech, Available from: http://www.intechopen.com/books/cognitive-maps/subject-formal-methodsbased-on-cognitive-maps-and-the-problem-of-risk-due-to-the-human-factor

\section{INTECH}

open science | open minds

\section{InTech Europe}

University Campus STeP Ri Slavka Krautzeka 83/A 51000 Rijeka, Croatia Phone: +385 (51) 770447 Fax: +385 (51) 686166 www.intechopen.com

\section{InTech China}

Unit 405, Office Block, Hotel Equatorial Shanghai No.65, Yan An Road (West), Shanghai, 200040, China 中国上海市延安西路65号上海国际贵都大饭店办公楼 405 单元 Phone: +86-21-62489820

Fax: $+86-21-62489821$ 
(C) 2010 The Author(s). Licensee IntechOpen. This chapter is distributed under the terms of the Creative Commons Attribution-NonCommercialShareAlike-3.0 License, which permits use, distribution and reproduction for non-commercial purposes, provided the original is properly cited and derivative works building on this content are distributed under the same license. 